\title{
ANNUAL R EPORT FOR \\ Environmental Management Science Program Project Number 86598 COUPLED FloW AND R EACTIVITY IN VARIABLy SATURATED POROUS MEDIA
}

Principal Investigator

Carl D. Palmer

Idaho National Engineering and

Environmental Laboratory

Idaho Falls, ID 83415

(208) 526-4478

palmcd@inel.gov
Co-Principal Investigators

Earl D. Mattson

Idaho National Engineering and

Environmental Laboratory

Idaho Falls, ID 83415

(208) 526-4084

matted@inel.gov

\author{
Robert W. Smith \\ University of Idaho-Idaho Falls \\ Idaho Falls, ID 83402 \\ (208) 282-7954 \\ smithbob@uidaho.edu
}

\section{RESEARCH OBJECTIVE}

Improved models of contaminant migration in heterogeneous, variably saturated porous media are required to better define the long-term stewardship requirements for U.S. Department of Energy (DOE) lands and to assist in the design of effective vadosezone barriers to contaminant migrations. The objective of our three-year project is to meet the DOE need by developing new experimental approaches to describe adsorption and transport of contaminants in heterogeneous, variably saturated media (i.e., the vadose zone). The research specifically addresses the behavior of strontium, a high priority DOE contaminant. However, the key benefit of this research is improved conceptual models of how all contaminants migrate through heterogeneous, variably-saturated, porous media. Research activities are driven by the hypothesis that the reactivity of variably saturated porous media is dependent on the moisture content of the medium and can be represented by a relatively simple function applicable over a range of scales, contaminants, and media. A

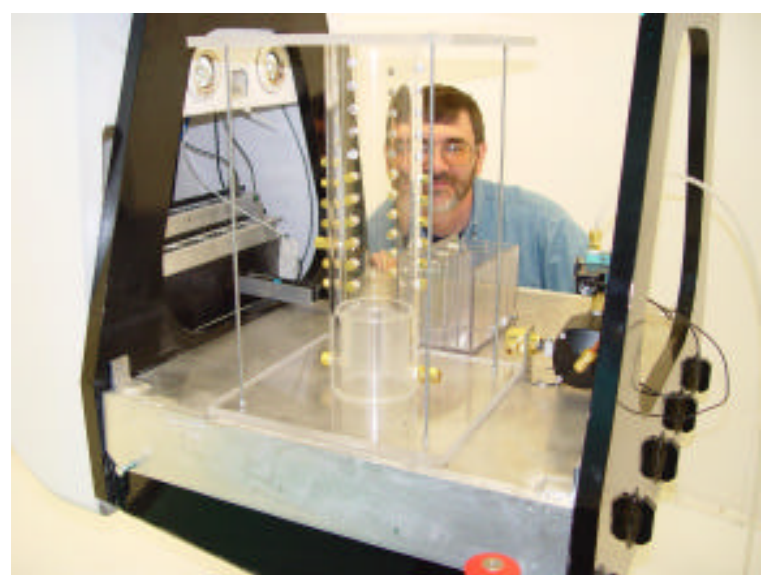

Figure 1. Basket (e.g., working platform) and experimental apparatus on the INEEL two-meter geocentrifuge. key and novel aspect of our research is the use of the 2-meter radius geocentrifuge capabilities at the Idaho National Engineering and Environmental Laboratory (INEEL) to conduct unsaturated reactive transport experiments (Figure 1). The experimental approach using the geocentrifuge provides data in a much shorter time period than conventional methods allowing us to complete more experiments and explore a wider range of moisture contents. The vadose zone research being done in this project will demonstrate the utility of environmental geocentrifuge experimental approaches and their applicability to DOE's vadose research needs. This report summarizes our progress as of June 2003 in the first year of a three-year project.

\section{RESEARCH PROGRESS AND IMPLICATIONS}

The objectives are being accomplished by conducting integrated conventional laboratory column experiments and geocentrifuge approaches to assess breakthrough curves for one- and two-dimensional systems with constructed heterogeneities under conditions of variable moisture content. In addition, we plan to conduct similar experiments using intact cores of natural soil formations. This report summarizes our progress as of June 15, 2003 and represents the first 9 months of a three-year project. Key activities to date include:

- $\quad$ Review of the theory of unsaturated flow in the geocentrifuge. Preliminary results suggest that a general theory of unsaturated fluid flow in a variably accelerated field such as found in a finite radius centrifuge is different from the scaling relationships that have previously been assumed and used in centrifuge studies. We are currently reviewing derivation of our equations and identifying limiting case relationships. We 
plan to present these results at a national meeting in the fall of 2003.

- Development of numerical tools. We are collaborating with Dr. Jirka Simunek to modify the Hydrus 1D and Hydrus 2D codes to account for variable acceleration field that is encountered on the geocentrifuge. We are currently familiarizing ourselves with these codes and using them in the design of our experiments. Additionally, these codes will be used in the analysis and interpretation of our experimental results. Pending the results of our theoretical studies, additional modifications of these codes may be required.

- Design of geocentrifuge experiments. Onedimensional geocentrifuge column experiments are being designed. Key experimental considerations that need to be addressed are fluid delivery systems, basic column design, measurement of soil moisture content and matric potential, inline measurement of specific conductance and specific ions, and collection of soil water samples. While such issues are well established for conventional laboratory experiments, there is little experience in identifying robust instrumentation that can be remotely controlled while in flight on the geocentrifuge. Much or our effort in the first year has been dedicated to resolving these issues.

\section{PLANNED ACTIVITIES}

We will complete design and testing of onedimensional geocentrifuge experimental systems by the end of FY-2003. Follow this, one-dimensional experiments on ostensibly homogeneous systems to determine the properties of the materials that will be used in the two-dimensional experiments will be conduct next year. In addition to geocentrifuge experiments, conventional column experiments using a vacuum chamber will be conducted to provide a direct comparison between the techniques. Both types of experiments will utilize conservative $\left(\mathrm{Cl}^{-}\right)$ and reactive tracers $\left(\mathrm{Sr}^{2+}, \mathrm{Zn}^{2+}, \mathrm{F}^{-}\right)$to obtain breakthrough curves as functions of moisture content. In addition, moisture content-matric potentialhydraulic conductivity relationships will be experimentally obtained. Our experimental results will be used to determine reactivity-moisture content relationships.
In addition to one-dimensional experiments, twodimensional tracer experiments will be conducted using the geocentrifuge and obviously heterogeneous materials. The type of heterogeneities consider will include: 1) systems with continuous alternating horizontal layers of low and high permeability; 2) systems with continuous alternating vertical bands of low and high permeability that can simulate vertical fractures (INEEL) or clastic dikes (Hanford); and 3) systems with discontinuous high permeability layers in a low permeability matrix or discontinuous low permeability layers in a high permeability matrix that can simulate clay lenses or the current conceptualization of the INEEL vadose zone. Although the experiments will examine idealizedheterogeneity, the heterogeneity patterns considered capture the essential features and characteristics of common types of heterogeneities observed in the subsurface at DOE sites. The two-dimensional experiments will be initiated in late winter 2004.

"Intact" sections of soil will be obtained for experiments in the geocentrifuge. We have initiated discussions with members of Phil Jardines's research group (support through EMSP project number 86911) and are planning a trip to Oak Ridge in late summer of 2003 to continue our discussions on collaborating in the collection of an intact core and the sharing of experimental results. We plan to conduct the intact section experiment in early FY 2005. In this experiment, the intact section will be loaded into the geocentrifuge and monitored in a manner similar to that used for the ideal heterogeneous packs described in the previous task. $\mathrm{Cl}^{-}, \mathrm{F}, \mathrm{Sr}^{2+}$ and $\mathrm{Zn}^{2+}$ will be run through the soil section, and breakthrough curves will be obtained for at least five different average water contents. The reactivity/moisture content obtained for this naturally heterogeneous soil will be compared to the relationships obtained from the twodimensional experiments using idealized heterogeneities to determine if the same general function is obtained. To test for potential anisotropy, we will conduct another experiment where flow is perpendicular to the first experiments. The data will be analyzed using the numerical tools described previously.

\section{INFORMATION ACCESS}

Discussions of our project were presented at the Geocentrifuge Workshop held at the Idaho National Engineering and Environmental Laboratory, Idaho Falls, ID, March 27-28, 2003. Information about the workshop and summaries of presentations can be 
found at www.inel.gov and search under "geocentrifuge".

A presentation of our project was given at the EMSP

PI meeting held at the Environmental Molecular

Sciences Laboratory at PNNL, May 6-8, 2003.

Information on presentation can be found at

www.pnl.gov/emsp/fy2003/presentations/palmer_carl

_86598.pdf 